\title{
Analytical interatomic bond-order potential for simulations of oxygen defects in iron
}

\section{Byggmästar, Jesper Johan André}

2019-05-29

Byggmästar , J J A , Nagel , M J , Albe , K , Henriksson, K O E \& Nordlund , K H 2019 , ' Analytical interatomic bond-order potential for simulations of oxygen defects in iron ', Journal of Physics. Condensed Matter , vol. 31 , no. 21 , 215401 . https://doi.org/10.1088/1361-648X/ab0931

http://hdl.handle.net/10138/301062

https://doi.org/10.1088/1361-648X/ab0931

acceptedVersion

Downloaded from Helda, University of Helsinki institutional repository.

This is an electronic reprint of the original article.

This reprint may differ from the original in pagination and typographic detail.

Please cite the original version. 
Preprint of article published by IOP Publishing Ltd

J. Phys.: Condens. Matter. 31215401 (2019)

https://doi.org/10.1088/1361-648X/ab0931

\title{
Analytical interatomic bond-order potential for simulations of oxygen defects in iron
}

\author{
J Byggmästar ${ }^{1}$, M Nagel $^{1}$, K Albe $^{2}$, K O E Henriksson ${ }^{1}$ \\ and $\mathbf{K}$ Nordlund $^{1}$ \\ 1 Department of Physics, P.O. Box 43, FI-00014 University of Helsinki, Finland \\ 2 Fachgebiet Materialmodellierung, Institut für Materialwissenschaft, Technische \\ Universität Darmstadt, Jovanka-Bontschits-Straße 2, D-64287 Darmstadt, \\ Germany \\ E-mail: jesper.byggmastar@helsinki.fi
}

\begin{abstract}
We present an analytical bond-order potential for the $\mathrm{Fe}-\mathrm{O}$ system, capable of reproducing the basic properties of wüstite as well as the energetics of oxygen impurities in $\alpha$-iron. The potential predicts binding energies of various small oxygen-vacancy clusters in $\alpha$-iron in good agreement with density functional theory results, and is therefore suitable for simulations of oxygen-based defects in iron. We apply the potential in simulations of the stability and structure of $\mathrm{Fe} / \mathrm{FeO}$ interfaces and $\mathrm{FeO}$ precipitates in iron, and observe that the shape of $\mathrm{FeO}$ precipitates can change due to formation of well-defined $\mathrm{Fe} / \mathrm{FeO}$ interfaces. The interface with crystalline Fe also ensures that the precipitates never become fully amorphous, no matter how small they are.
\end{abstract}

Keywords: interatomic potential, iron, oxygen, molecular dynamics 


\section{Introduction}

Iron and different types of steels belong to the most widely used materials in modern society. New types of radiation-resistant iron alloys, such as oxidedispersion-strengthened (ODS) steels, are continuously being investigated as possible candidates for future fusion and fission reactor materials [1]. In ODS steels, small nano-sized Y-Ti-O particles are allowed to precipitate within the steel matrix, leading to enhanced high-temperature mechanical properties and radiation tolerance [1, 2. The compositions and crystal structures of the oxide nanoparticles, as well as the interfaces they form with the steel matrix, are still actively debated research topics [2, 3, 4, The mobility and clustering of oxygen in the steel is essential for understanding the precipitation and growth process of the nanoparticles, a topic that can be aided by insights from atomistic simulations.

Much of what is known about the behaviour of oxygen in iron has been obtained by density functional theory (DFT) calculations [5, 6, 7, 8, 9, 10. However, modelling of precipitation growth or long-range diffusion and clustering of point defects, for example, require computationally more efficient methods. Larger scale atomistic simulations, such as molecular dynamics (MD) or various Monte Carlo methods, rely on the use of an interatomic potential for describing the interactions between the individual atoms. For the $\mathrm{Fe}-\mathrm{O}$ system, Aryanpour et al. have parametrised a reactive force field (ReaxFF) that is capable of reproducing the thermodynamics of the common iron oxides [11. However, no FeO many-body potential has to our knowledge been fitted primarily for large-scale simulations of oxygen impurities in iron.

In this study, we present an analytical bond-order potential $(\mathrm{ABOP})$ for the $\mathrm{Fe}-\mathrm{O}$ system. Existing parameter sets are used for the elemental interactions $\mathrm{Fe}-\mathrm{Fe}$ [12, 13, and $\mathrm{O}-\mathrm{O}$ [14, and only the $\mathrm{Fe}-\mathrm{O}$ parameter set is developed here. The Fe-Fe ABOP, providing the basis of the $\mathrm{Fe}-\mathrm{O}$ parametrisation developed in this study, correctly reproduces the $\alpha-\gamma-\delta$ phase transition and has been extensively applied in simulations of e.g. collision cascades [13], stretching of nanowires [15, and as a basis for other iron compound potentials [16, 17, 18, 19.

The rest of the article is structured as follows. In section 2, the functional form of the potential is briefly presented. The properties of the stable iron oxides and oxygen solutes in iron are then reviewed to gather a database for fitting and testing of the $\mathrm{Fe}-$ $\mathrm{O}$ potential. In section 3, we discuss and compare the fitted properties to experimental and ab initio data. We then present results of further testing of the potential by calculating properties not included in the fitting process. Finally, we apply the potential in a study of $\mathrm{FeO} / \mathrm{Fe}$ interfaces and $\mathrm{FeO}$ nanoparticles embedded in iron.

\section{Methods}

\subsection{Computational details}

The interatomic potential was numerically fitted using the code TULIP [20. Molecular dynamics (MD) simulations were carried out with LAMMPS 21] and PARCAS [22, 23. Structure optimisations, defect energies, and phonon dispersions were calculated using ASE [24] and LAMMPS.

Density functional theory (DFT) calculations were carried out using VASP [25]. The implementation uses the projector augmented-wave method [26] with the GGA-PBE exchange-correlation functional [27]. The calculations were done using spin-polarisation. Careful convergence checks were performed to find suitable energy cutoffs and $k$-point densities. For the final calculations, we used a $17 \times 17 \times 17 k$-point mesh for the $\mathrm{FeO}$ primitive unit cell The plane-wave cutoff energy was $800 \mathrm{eV}$. A Gaussian smearing of $0.2 \mathrm{eV}$ was applied to help the convergence.

\subsection{Potential formalism}

The concept of bond order in interatomic potentials as discussed by Abell [28, was first utilised in empirical many-body potentials by Tersoff [29, 30 for covalently bonded materials 31, 32. The analytical bond-order potential (ABOP) formalism used in this work is similar to the Tersoff potential, but slightly rewritten and extended in a form similar to that of Brenner [33]. Despite being originally developed for covalently bonded materials, the bond-order potentials are fundamentally related to the embedded atom method potentials 34, 35] developed for metals [36. Consequently, the $\mathrm{ABOP}$ has, in addition to purely covalent materials, also been successfully applied to a wide range of differently bonded compounds, including 
partly ionic metal oxides [14, 37. The ABOP formalism has been presented in detail previously [38, 39, and is only shortly summarised below.

The total potential energy in the ABOP is given as a function of the interatomic distance $r_{i j}$ by

$V=\sum_{i} \sum_{j>i} V_{i j}=\sum_{i} \sum_{j>i} f_{\mathrm{C}}\left(r_{i j}\right)\left[V_{\mathrm{R}}\left(r_{i j}\right)-\bar{b}_{i j} V_{\mathrm{A}}\left(r_{i j}\right)\right]$,

where the repulsive and attractive pair functions are the Morse-like potentials

$V_{\mathrm{R}}\left(r_{i j}\right)=\frac{D_{0}}{S-1} \exp \left[-\beta \sqrt{2 S}\left(r_{i j}-r_{0}\right)\right]$,

$V_{\mathrm{A}}\left(r_{i j}\right)=\frac{S D_{0}}{S-1} \exp \left[-\beta \sqrt{2 / S}\left(r_{i j}-r_{0}\right)\right]$.

$D_{0}$ and $r_{0}$ are the dimer bond energy and length, and $\beta$ and $S$ are fitting parameters controlling the shape of the pair potential. The interaction range is restricted by the cutoff function, which drives the potential smoothly to zero in the range $R-D$ to $R+D$, according to

$f_{\mathrm{C}}(r)= \begin{cases}1, & r \leq R-D \\ \frac{1}{2}-\frac{1}{2} \sin \left[\frac{\pi}{2 D}(r-R)\right], & |R-r|<D(4) \\ 0, & r \geq R+D .\end{cases}$

The bond-order function incorporates the many-body dependence and is written in the symmetric form

$\bar{b}_{i j}=\frac{b_{i j}+b_{j i}}{2}$,

where

$b_{i j}=\left(1+\chi_{i j}\right)^{-1 / 2}$.

Furthermore, the effective coordination number is calculated as

$\chi_{i j}=\sum_{k(\neq i, j)} f_{\mathrm{C}}\left(r_{i k}\right) g_{i k}\left(\theta_{i j k}\right) \omega_{i j k} \exp \left[\alpha_{i j k}\left(r_{i j}-r_{i k}\right)\right],(7)$

where the angular function is given by

$g_{i k}\left(\theta_{i j k}\right)=\gamma_{i k}\left[1+\frac{c_{i k}^{2}}{d_{i k}^{2}}-\frac{c_{i k}^{2}}{d_{i k}^{2}+\left(h_{i k}+\cos \theta_{i j k}\right)^{2}}\right]$.

The sensitivity of the angular dependence is tuned by the parameters $c_{i k}$ and $d_{i k}$. The optimal angle $\theta_{i j k}$ between atoms $i j k$ is set by the parameter $h_{i k}$, at which the angular function reaches a minimum (given by the value of $\gamma_{i k}$ ). The three-body $\alpha_{i j k}$ parameters are in some versions of the potential replaced by the two-body parameter $2 \mu_{i k}$, which in many cases is set equal to zero. Using non-zero $\alpha_{i j k}$ values enables the dependence on the relative bond distances between the three atoms $i j k$, which for some compounds is desirable. Similarly, $\omega_{i j k}$ are often set equal to one for all atom triplets $i j k$, but can be modified if rescaling the two-body $\gamma_{i k}$ parameter for specific $i j k$ interactions is necessary. The formalism does not explicitly include any magnetic interactions, but properties sensitive to magnetism can still be reproduced by careful tuning of the above parameters, as shown in Ref. [12]. Recently, there has also been progress on including magnetic contributions in bond-order potentials for iron [40, 41].

In simulations of high-energy events, such as collision cascades, the repulsive part of the potential must be modified to give a more accurate behaviour at short interatomic distances. The standard approach is to join the universal repulsive Ziegler-BiersackLittmark potential [42], $V_{\mathrm{ZBL}}\left(r_{i j}\right)$, with the original potential $V_{i j}$ as

$V_{i j}^{\prime}=F\left(r_{i j}\right) V_{i j}+\left[1-F\left(r_{i j}\right)\right] V_{\mathrm{ZBL}}$.

Here, $F(r)$ is the Fermi function

$F(r)=\frac{1}{1+\exp \left[-b_{\mathrm{f}}\left(r-r_{\mathrm{f}}\right)\right]}$.

The parameters $b_{\mathrm{f}}$ and $r_{\mathrm{f}}$ are chosen so that the equilibrium properties of all relevant structures remain nearly unchanged.

\subsection{Fitting database}

2.3.1. Iron oxides Iron oxides occur in nature mainly in three stable mineral forms; magnetite, wüstite, and hematite. At low temperatures and oxygen concentrations, the oxygen crystallises into magnetite $\left(\mathrm{Fe}_{3} \mathrm{O}_{4}\right.$, space group $F d \overline{3} m$, no. 227) mixed with $\alpha$ Fe. Wüstite $\left(\mathrm{Fe}_{1-x} \mathrm{O}\right.$, space group $F m \overline{3} m$, no. 225$)$ is stable at temperatures above around $840 \mathrm{~K}$. Hematite $\left(\mathrm{Fe}_{2} \mathrm{O}_{3}\right.$, space group $R \overline{3} c$, no. 167) crystallises in oxygen-rich environments 43 .

Wüstite $\left(\mathrm{Fe}_{1-x} \mathrm{O}\right)$ crystallises into an irondeficient rocksalt structure. The value of $1-x$ in $\mathrm{Fe}_{1-x} \mathrm{O}$ ranges from 0.83 to 0.95 [44]. Stoichiometric $\mathrm{FeO}$ is not stable at low pressures. The lattice parameter is $4.28-4.33 \AA$, depending on the iron vacancy concentration 44. The effect of nonstoichiometry, i.e. the deviation of $x$ from zero, has been shown to strongly affect the elastic properties. The bulk modulus of wüstite has been extensively studied experimentally and is fairly insensitive to the non-stoichiometry in the range $0.90<1-x<0.98$, which represent typical experimental samples. In that range, the reported values for the bulk modulus are around 144-152 GPa [45]. However, motivated by much higher theoretically predicted values for stoichiometric $\mathrm{FeO}$, Zhang found that samples with 1$x \approx 0.99$ have a bulk modulus significantly higher (175 $\mathrm{GPa}$ ) than for typical values of $1-x$ [45]. The effects of non-stoichiometry on the elasticity is important to keep in mind when gathering experimental reference data for the potential fitting database, as we only consider stoichiometric $\mathrm{FeO}$ in the numerical fitting. As a reference value for the bulk modulus of ideal $\mathrm{FeO}$, we use the value reported by Zhang for $\mathrm{Fe}_{0.99} \mathrm{O}$. However, the elastic constants used as reference values 
in the fitting, and cited in table 1. were obtained from experimental studies with sample compositions in the range $\mathrm{Fe}_{0.90-0.95} \mathrm{O}$ [46], which should be taken into consideration when comparing with the values predicted by the $\mathrm{ABOP}$ for stoichiometric $\mathrm{FeO}$.

Magnetite $\left(\mathrm{Fe}_{3} \mathrm{O}_{4}\right)$ crystallises as the inverse spinel structure. Oxygen atoms are ordered in a facecentred cubic structure, with $1 / 2$ of the octahedral and $1 / 8$ of the tetrahedral sites filled with Fe atoms. Experimental results for the structural and elastic properties are available from single crystal $\mathrm{Fe}_{3} \mathrm{O}_{4}$ studies [47, 48].

Hematite $\left(\alpha-\mathrm{Fe}_{2} \mathrm{O}_{3}\right)$ has the same crystal structure as Corundum $\left(\mathrm{Al}_{2} \mathrm{O}_{3}\right)$. The oxygen atoms are ordered in a slightly distorted hexagonal close-packed structure, with $2 / 3$ of the octahedral sites filled with $\mathrm{Fe}$ atoms. Hematite has been extensively studied experimentally and reference values for the structural properties are readily available [49, 50, 51].

Reference values for the cohesive or formation energies of the different phases can be obtained from the experimental enthalpies of formation (measured at room temperatures). At normal pressures, the enthalpy is approximately the same as the internal potential energy, $H=U+P V \approx U$. In the formation of a compound $A_{m} B_{n}$, the relation between the cohesive and formation energies is

$E_{\mathrm{f}}=E_{\mathrm{p}}\left(A_{m} B_{n}\right)-m E_{\mathrm{coh}}(A)-n E_{\mathrm{coh}}(B)$,

where $E_{\mathrm{p}}\left(A_{m} B_{n}\right)$ is the potential energy of $A_{m} B_{n}$, and $E_{\text {coh }}$ the cohesive energies of the ground states of $A$ and $B$. The formation and cohesive energies for the different iron oxides, together with the other structural and mechanical properties, are given in table 1 and used in the fitting of the potential.

2.3.2. Oxygen defects in $\alpha-F e$ First-principles calculations of oxygen defects in iron have been carried out in a number of studies [52, 7, 53, 54, 5, 6]. A database for defect properties for fitting and testing of the $\mathrm{Fe}-\mathrm{O}$ potential can therefore be constructed from the literature.

For oxygen atoms in $\alpha$-Fe, the octahedral interstitial site is preferred over tetrahedral sites, with both being strongly favoured over a substitutional lattice site. The formation energies for the different oxygen point defect sites, compiled from the literature, are given in table 1 . Oxygen has a low solubility in defect-free iron, but it has been shown that the presence of vacancies in the iron lattice strongly affects the solubility and mobility of oxygen [52. Compared to other solutes, oxygen atoms form strong bonds with nearby vacancies in iron. The vacancy concentration therefore essentially controls the solubility of oxygen, allowing the oxygen concentration to reach that of the vacancies by forming oxygenvacancy $(\mathrm{O}-V)$ bonds $[52$. The strong $\mathrm{O}-V$ bonds also significantly reduce the mobility of interstitial oxygen solutes. Octahedral oxygen atoms in a vacancy-free iron lattice migrate to another octahedral site, passing through the tetrahedral site with a migration barrier of $0.48-0.60 \mathrm{eV}$ [52, 7, 53]. If, however, the oxygen atom has a nearest-neighbour vacancy, the $\mathrm{O}-V$ pair has been shown to migrate together as a pair in a twostep process [52. The vacancy then first moves to a nearby lattice site, after which the oxygen atom quickly reconnects with the vacancy at its nearest-neighbour octahedral site. The energy barrier for this two-step $\mathrm{O}-V$ migration is significantly higher $(1.55 \mathrm{eV}[52])$ than for single oxygen migration in a perfect iron lattice. The binding energy of the $\mathrm{O}-V$ pair has also been calculated for bond lengths longer than the first nearest-neighbour distance, and was found to be significantly strong also for second nearest-neighbour distances [7, 53]. Additionally, the binding energies and diffusion mechanisms for clusters with multiple vacancies or oxygen atoms $\left(V_{n} \mathrm{O}_{m}\right)$ in iron have been recently studied in first-principles calculations [5, 6.

The substantial amount of available experimental and ab initio data provides a good database for guiding the fitting of the ABOP parameters. The data used in the fitting process are given in table 1 . The parameters of the $\mathrm{Fe}-\mathrm{Fe}$ and $\mathrm{O}-\mathrm{O}$ potentials were kept fixed according to the values given in [12, 13, 14].

\section{Results and discussion}

\subsection{Fitted properties}

The $\mathrm{Fe}-\mathrm{O}$ parameters were fitted with the main goal of achieving a good description of the oxygen point defects in $\alpha$-Fe. The predicted properties of the different iron oxides and single oxygen defects with the best parameter fit are given in table 1 . The fitted parameters are given in tables 23.

The formation energies of the single oxygen defects in $\alpha$-Fe and the structural properties of $\mathrm{FeO}$ (wüstite) are well reproduced by the ABOP. Due to the experimentally observed differences in the properties of different degrees of non-stoichiometry in $\mathrm{Fe}_{1-x} \mathrm{O}$, as discussed previously, we calculated the lattice constant and formation energy of ideal FeO with DFT. The obtained lattice constant and formation energy of $\mathrm{FeO}$ are both the same as the experimental $\mathrm{Fe}_{1-x} \mathrm{O}$ data, which could therefore be used as fitting targets for $\mathrm{FeO}$.

A good description of the oxygen defects and the wüstite phase was favoured over the more oxygenrich magnetite and hematite structures. The latter structures proved to be challenging to stabilise within the ABOP, partly due to the range of the underlying $\mathrm{Fe}-\mathrm{Fe}$ potential. In all three iron oxides, the 
shortest $\mathrm{Fe}-\mathrm{Fe}$ bonds are within the cutoff radius of the Fe potential, so that pure iron interactions always contribute to the total potential energy of the oxide structures. We found that these contributions are disproportionally different between the oxide structures. The potential energy contribution from $\mathrm{Fe}-\mathrm{Fe}$ bonds in the high-coordinated wüstite structure is significantly greater than in the lower-coordinated (in terms of $\mathrm{Fe}-\mathrm{Fe}$ bonds) magnetite and hematite structures. The consequence is that the bond energies from $\mathrm{Fe}-\mathrm{O}$ interactions required to reproduce the experimental cohesive energies also become disproportionally different, so that, for example, fitting the ABOP to the dimer and wüstite energies and bond lengths would severely underestimate the $\mathrm{Fe}-\mathrm{O}$ bond strengths in hematite and magnetite, and vice versa. As a compromise to partly compensate for this effect, the bond energy of the $\mathrm{Fe}-\mathrm{O}$ dimer was allowed to increase to values well above the reference value during the fitting routine. Additionally, the three-body $\alpha_{i j k}$ and $\omega_{i j k}$ were included in the fitting process to partly compensate for the effects of the $\mathrm{Fe}-\mathrm{Fe}$ interactions. Nevertheless, achieving a good description of the oxygen-rich magnetite and hematite phases was not possible (without modifying the pure $\mathrm{Fe}-\mathrm{Fe}$ potential), but was accepted in favour of good properties for oxygen-based defect structures in pure iron. For more details about the fitting strategy, see Ref. [39].

The cutoff value of the $\mathrm{Fe}-\mathrm{O}$ interactions was chosen to be between the first and second nearest $\mathrm{Fe}-\mathrm{O}$ neighbours in wüstite. In both hematite and magnetite, this range includes the second nearest neighbours. All other parameters were kept as free parameters during the fitting. Due to the strongly overestimated dimer energy, it is crucial to make sure that the lower-coordinated structures are not also incorrectly favoured over the $\mathrm{NaCl}$ structure for $\mathrm{FeO}$ (wüstite). Calculating the energies of the hypothetical $\mathrm{ZnS}$, wurtzite, $\mathrm{CsCl}$, and $\mathrm{NiAs}$ structures for $\mathrm{FeO}$ revealed that all were around a minimum of $0.1 \mathrm{eV}$ or higher in cohesive energy, correctly making wüstite the ground state of $\mathrm{FeO}$. This was confirmed in an MD simulation, where crystallisation into the rocksalt structure was observed after slowly cooling a molten $\mathrm{FeO}$ system to below the melting point. The properties of magnetite $\left(\mathrm{Fe}_{3} \mathrm{O}_{4}\right)$ and hematite $\left(\mathrm{Fe}_{2} \mathrm{O}_{3}\right)$ are listed in table 1 to show that the ABOP predicts reasonable energetics for different iron oxide stoichiometries, but both structures are unstable in the ABOP and collapse into amorphous phases already at room temperature. The ABOP is therefore not suitable for simulations involving the magnetite or hematite phases.
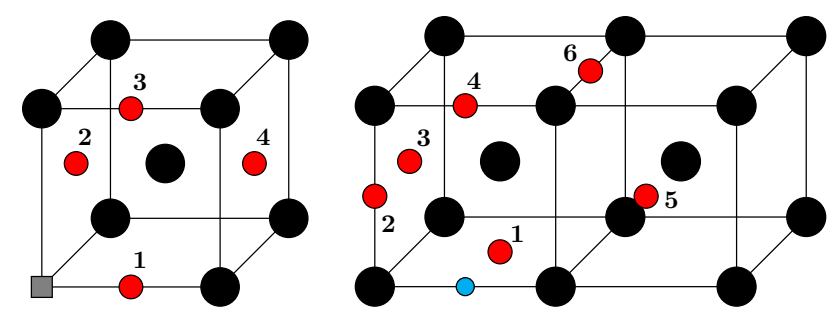

Figure 1. Left: nearest octahedral sites (red atoms) of a vacancy (grey square) in bcc. Right: nearest octahedral neighbours of another octahedral site (blue atom). Black atoms are bcc lattice sites.

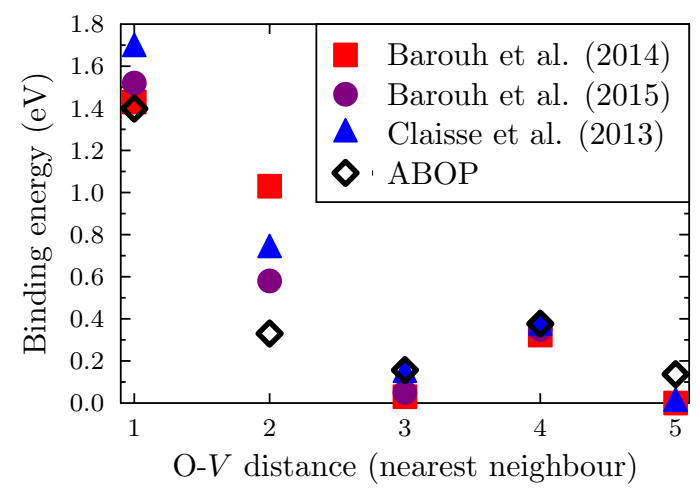

Figure 2. Binding energies between an octahedral oxygen atom and a vacancy in bcc iron predicted by ABOP, and compared with published DFT results [5, 6, 7].

\subsection{Tested properties}

3.2.1. Oxygen-vacancy defect clusters in $\alpha$-Fe In order to test the ABOP for atomic geometries not considered in the fitting process, binding energies of various defect configurations, beyond the single oxygen defects in $\alpha$-Fe, were calculated and compared with published DFT results. The binding energy between two defect clusters $A_{n} B_{m}$ and $A_{n^{\prime}} B_{m^{\prime}}$ is defined as

$E_{\mathrm{b}}=E\left(A_{n} B_{m}\right)+E\left(A_{n^{\prime}} B_{m^{\prime}}\right)-E\left(A_{n+n^{\prime}} B_{m+m^{\prime}}\right)-E_{0},(12)$

where $E\left(A_{n} B_{m}\right), E\left(A_{n^{\prime}} B_{m^{\prime}}\right), E\left(A_{n+n^{\prime}} B_{m+m^{\prime}}\right)$ are the potential energies of the systems containing the given clusters, and $E_{0}$ is the energy of the defect-free bulk. Positive binding energies correspond to an attraction between the defect clusters. The nearest interstitial (octahedral) neighbours of a vacancy in bcc iron, as well as nearest octahedral-octahedral neighbours are illustrated in figure 1 .

Figures 25 show the binding energies of different defect configurations as predicted by ABOP. The defects were added to systems of 250 bcc Fe atoms and minimised in energy at zero pressure to get the total energies needed in Equation 12. Tests with larger bcc systems produced identical results to within about a percent. The binding energies for the $\mathrm{O}-V$ pair 
Table 1. Experimental and DFT literature values for properties of different iron oxides and oxygen defects used as the fitting database, compared with values predicted by the ABOP parametrisation. $r_{0}$ : dimer bond length $(\AA), E_{\mathrm{b}}$ : dimer bond energy (eV), $a, c$ : lattice constants $(\AA), E_{\mathrm{f}}$ : formation energy $(\mathrm{eV} / \mathrm{atom}), E_{\text {coh }}$ : cohesive energy $(\mathrm{eV} / \mathrm{atom}), B$ : bulk modulus (GPa), and $c_{i j}$ : elastic constants $(\mathrm{GPa}) . z_{\mathrm{Fe}}$ and $x_{\mathrm{O}}$ are the internal coordinates of the $\mathrm{Fe}_{2} \mathrm{O}_{3}$ crystal structure.

\begin{tabular}{lccc} 
& DFT & Experiment & ABOP \\
\hline Fe-O dimer & & & \\
\hline$r_{0}$ & $1.614^{\mathrm{a}}$ & $1.619^{\mathrm{b}}$ & 1.58 \\
$E_{\mathrm{b}}$ & $5.47^{\mathrm{a}}$ & $4.13-4.21^{\mathrm{b}}$ & 7.93
\end{tabular}

FeO wüstite $(F m \overline{3} m$, no. 225$)$

\begin{tabular}{|c|c|c|c|}
\hline$a$ & $4.31^{\mathrm{a}}$ & $4.31^{\mathrm{c}}$ & 4.31 \\
\hline$E_{\mathrm{f}}$ & $-1.40^{\mathrm{a}}$ & $-1.41^{\mathrm{d}}$ & -1.46 \\
\hline$E_{\text {coh }}$ & $-4.83^{\mathrm{a}}$ & -4.84 & -4.84 \\
\hline$B$ & & $175^{\mathrm{c}}$ & 183 \\
\hline$c_{11}$ & & $217-246^{\mathrm{e}}$ & 399 \\
\hline$c_{12}$ & & $121-149^{\mathrm{e}}$ & 76 \\
\hline$c_{44}$ & & $44-50^{\mathrm{e}}$ & 49 \\
\hline \multicolumn{4}{|c|}{$\mathrm{Fe}_{3} \mathrm{O}_{4}$ magnetite $(F d \overline{3} m, \text { no. } 227)^{*}$} \\
\hline$a$ & & $8.39^{\mathrm{f}}$ & 8.58 \\
\hline$E_{\mathrm{f}}$ & & $-1.66^{\mathrm{d}}$ & -1.44 \\
\hline$E_{\mathrm{coh}}$ & & -4.96 & -4.70 \\
\hline$B$ & & $185.7^{\mathrm{g}}$ & 97 \\
\hline \multicolumn{4}{|c|}{$\mathrm{Fe}_{2} \mathrm{O}_{3}$ hematite $(R \overline{3} c, \text { no. } 167)^{*}$} \\
\hline$a$ & & $5.035^{\mathrm{g}}$ & 5.17 \\
\hline$c$ & & $13.747^{\mathrm{g}}$ & 12.80 \\
\hline$z_{\mathrm{Fe}}$ & & $0.3553^{\mathrm{g}}$ & 0.377 \\
\hline$x_{\mathrm{O}}$ & & $0.3056^{\mathrm{g}}$ & 0.255 \\
\hline$E_{\mathrm{f}}$ & & $-1.71^{\mathrm{d}}$ & -1.49 \\
\hline$E_{\mathrm{coh}}$ & & -4.97 & -4.72 \\
\hline$B$ & & $199^{\mathrm{h}} 231^{\mathrm{i}}$ & 235 \\
\hline$E_{\mathrm{f}}($ octahedral $\mathrm{O}$ in $\alpha$-Fe $)$ & $-0.38^{\mathrm{j}}$ & & -0.38 \\
\hline$E_{\mathrm{f}}($ tetrahedral $\mathrm{O}$ in $\alpha$-Fe $)$ & $0.13^{\mathrm{j}}$ & & 0.13 \\
\hline$E_{\mathrm{f}}($ substitutional $\mathrm{O}$ in $\alpha-\mathrm{Fe})$ & $1.06^{\mathrm{k}}, 1.28^{\mathrm{k}}$ & & 1.22 \\
\hline
\end{tabular}

${ }^{\mathrm{a}}$ This work ${ }^{\mathrm{b}}$ Ref. [55] $\quad{ }^{\mathrm{c}}$ Ref. [45] ${ }^{\mathrm{d}}$ Ref. [56]

${ }^{\mathrm{e}}$ Ref. [46] and references therein ${ }^{\mathrm{f}}$ Ref. [4]

${ }^{\mathrm{g}}$ Ref. [4] ${ }^{\mathrm{h}}$ Ref. [50] $\quad{ }^{\mathrm{i}}$ Ref. [51] ${ }^{\mathrm{j}}$ Ref. [7] ${ }^{\mathrm{k}}$ Calculated using the difference between $E_{\mathrm{f}}^{\text {sub }}$ and $E_{\mathrm{f}}^{\text {octa }}$ from Ref. [5] and Ref. [57] compared with the $E_{\mathrm{f}}^{\text {octa }}$ from Ref. [7] used above. ${ }^{*}$ Unstable in the ABOP. 
Table 2. Parameters for the $\mathrm{Fe}-\mathrm{O}$ ABOP. The $\mathrm{Fe}-\mathrm{Fe}$ and $\mathrm{O}-\mathrm{O}$ parameters are taken from earlier studies, and the $\mathrm{Fe}-$ $\mathrm{O}$ parameters are developed in this study. The $\alpha_{i j k}$ and $\omega_{i j k}$ parameters are given in table 3

\begin{tabular}{llll}
\hline & $\mathrm{Fe}-\mathrm{Fe}[12,13]$ & $\mathrm{O}-\mathrm{O}[14]$ & $\mathrm{Fe}-\mathrm{O}$ \\
\hline$D_{0}$ & 1.5 & 5.166 & 7.9851367774 \\
$r_{0}$ & 2.29 & 1.2075 & 1.5555688567 \\
$\beta$ & 1.4 & 2.3090 & 1.2135035992 \\
$S$ & 2.0693109 & 1.3864 & 2.8735224898 \\
$\gamma$ & 0.0115751 & 0.82595 & 1.1742630926 \\
$c$ & 1.2898716 & 0.035608 & 19.8656293772 \\
$d$ & 0.3413219 & 0.046496 & 4.5750149838 \\
$h$ & -0.26 & 0.45056 & 0.0904310711 \\
$R$ & 3.15 & 2.1 & 3.15 \\
$D$ & 0.2 & 0.2 & 0.15 \\
$b_{\mathrm{f}}$ & 2.9 & 12.0 & 10.0 \\
$r_{\mathrm{f}}$ & 0.95 & 0.5 & 1.0 \\
$\alpha$ & 0.0 & 0.0 & Table 3 \\
$\omega$ & 1.0 & 1.0 & Table \\
\hline
\end{tabular}

Table 3. The $\alpha_{i j k}$ and $\omega_{i j k}$ parameters for the Fe-O ABOP.

\begin{tabular}{ll} 
& $\mathrm{Fe}-\mathrm{O}$ \\
\hline$\alpha_{\mathrm{Fe}-\mathrm{Fe}-\mathrm{O}}$ & 1.0854004606 \\
$\alpha_{\mathrm{Fe}-\mathrm{O}-\mathrm{Fe}}$ & 0.3316469057 \\
$\alpha_{\mathrm{Fe}-\mathrm{O}-\mathrm{O}}$ & 3.4803491740 \\
$\alpha_{\mathrm{O}-\mathrm{Fe}-\mathrm{Fe}}$ & 1.0361635661 \\
$\alpha_{\mathrm{O}-\mathrm{Fe}-\mathrm{O}}$ & 0.7546312148 \\
$\alpha_{\mathrm{O}-\mathrm{O}-\mathrm{Fe}}$ & 0.001 \\
$\omega_{\mathrm{Fe}-\mathrm{Fe}-\mathrm{O}}$ & 0.9201732967 \\
$\omega_{\mathrm{Fe}-\mathrm{O}-\mathrm{Fe}}$ & 0.6842003046 \\
$\omega_{\mathrm{Fe}-\mathrm{O}-\mathrm{O}}$ & 1.0988780255 \\
$\omega_{\mathrm{O}-\mathrm{Fe}-\mathrm{Fe}}$ & 0.9823723952 \\
$\omega_{\mathrm{O}-\mathrm{Fe}-\mathrm{O}}$ & 1.0 \\
$\omega_{\mathrm{O}-\mathrm{O}-\mathrm{Fe}}$ & 1.0 \\
\hline
\end{tabular}

at different nearest neighbour (NN) separations are compared to three different DFT studies in figure 2 . The ABOP predicts a binding energy within $0.1-$ $0.2 \mathrm{eV}$ of the DFT values for the most stable (the nearest neighbour) $\mathrm{O}-V$ bond. Similar agreement is seen at longer $\mathrm{O}-V$ bonds, except for the second nearest neighbour (2NN) case, where the binding energy is underestimated compared to all DFT studies. However, the reference values for the $2 \mathrm{NN} \mathrm{O}-V$ pair are also fairly spread out, as opposed to the other NN distances.

Figure 3 shows the $\mathrm{O}-\mathrm{O}$ binding energies at different NN distances. The energies predicted by the ABOP follow a similar trend as the DFT results, but are consistently overestimated by about $0.2-0.4 \mathrm{eV}$. The $1 \mathrm{NN} \mathrm{O}-\mathrm{O}$ configuration (i.e. two octahedral $\mathrm{O}$

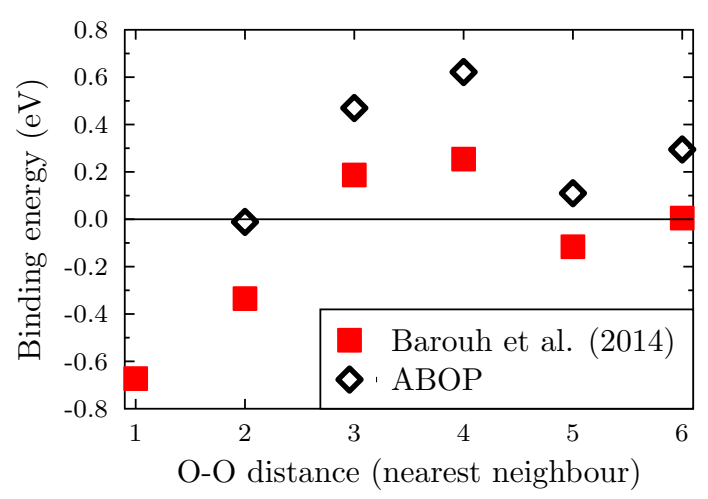

Figure 3. Binding energies between two octahedral oxygen atoms in bcc iron predicted by ABOP, and compared with published DFT results [5].

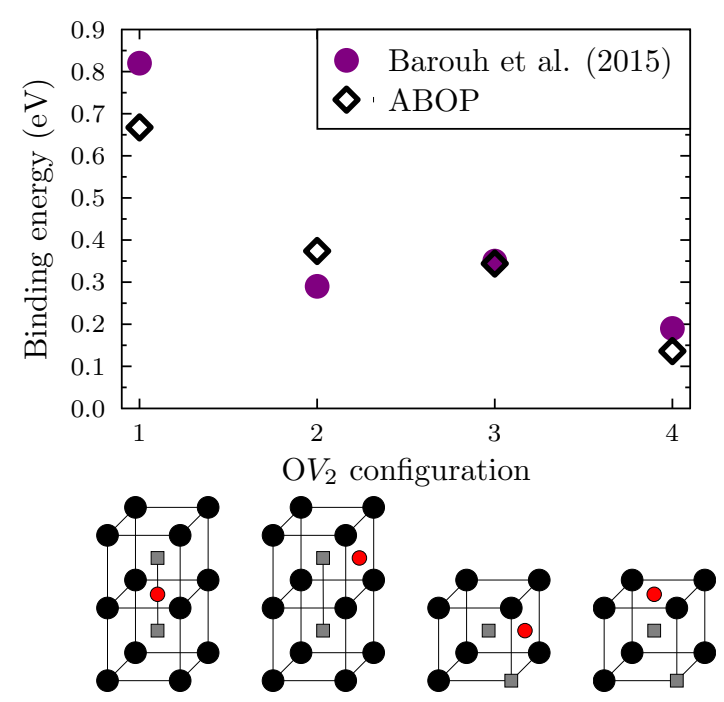

Figure 4. Binding energies for different $\mathrm{O} V_{2}$ configurations in bcc iron predicted by the ABOP and compared with DFT results [6]. The binding energies are calculated as the binding between a vacancy and an $\mathrm{O}-V$ pair according to Equation 12 In the illustrations of the defect sites, red circles represent oxygen, grey squares are vacancies, and black circles are iron atoms.

atoms half a lattice constant away from each other, see figure 1), is not stable in the $\mathrm{ABOP}$ and relaxes to the $4 \mathrm{NN}$ configuration (in which one lattice constant separates the $\mathrm{O}$ atoms). The binding energy for the $1 \mathrm{NN}$ case obtained by Barouh et al. is also strongly negative, meaning the interaction between the $\mathrm{O}$ interstitials is non-bonding.

Figure 4 shows the binding energies between different $\mathrm{OV}_{2}$ configurations in iron as obtained with the ABOP and compared with DFT results by Barouh et al. 6]. The binding energies are calculated as the binding between a vacancy and an $\mathrm{O}-V$ pair according 


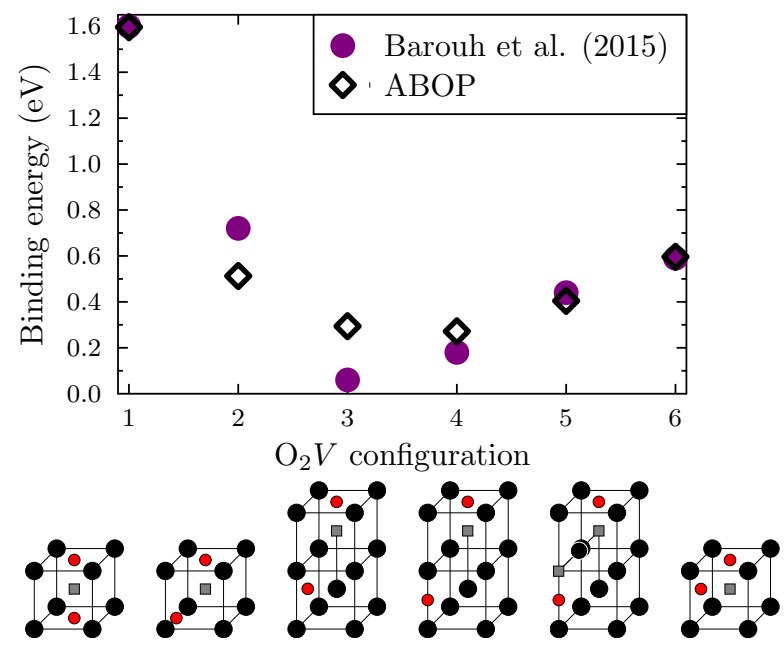

Figure 5. Binding energies for different $\mathrm{O}_{2} \mathrm{~V}$ configurations in bcc iron predicted by the ABOP and compared with DFT results [6]. The binding energies are calculated as the binding between an oxygen atom and an $\mathrm{O}-V$ pair according to Equation 12 In the illustrations of the defect sites, red circles represent oxygen, grey squares are vacancies, and black circles are iron atoms.

to Equation 12. The unrelaxed positions of the defects are also shown in figure 4. The overall agreement between the ABOP and the DFT results is good. The binding energy for the most stable $\mathrm{OV}_{2}$ configuration is underestimated by about $0.15 \mathrm{eV}$. The other calculated energies are within the same range from the DFT values, although the order of stability is reversed for configurations two and three.

For the $\mathrm{O}_{2} V$ defects in figure 5 , the binding energy for the most stable configuration is in perfect agreement with the DFT result. The other cases are within about $0.2 \mathrm{eV}$ of the DFT values and follow a similar trend. Configuration three is overestimated by about $0.25 \mathrm{eV}$ compared to the DFT value and, in contrast to DFT, more stable than configuration four. However, the differences in stability of the different defect configurations are small. The ABOP correctly predicts that the binding energy for the favoured case, configuration one, is at the same level $(1.6 \mathrm{eV})$, or even slightly higher, than the binding energy of the most stable $\mathrm{O} V$ case $(1 \mathrm{NN})$.

Oxygen interstitials in vacancy-free $\alpha$-Fe in the ABOP migrate between adjacent octahedral sites with a migration barrier of $0.55 \mathrm{eV}$, in agreement with DFT (0.48-0.60 eV [52, 7, [53]). The above results show that the $\mathrm{ABOP}$ is also able to reproduce the effects of vacancies in the vicinity of oxygen interstitials. More specifically, that vacancies act as strong traps for migrating oxygen interstitials [52]. Most importantly, the ABOP reproduces the most stable oxygen-vacancy configurations $\left(\mathrm{O}_{2} V\right.$ and $1 \mathrm{NN}$ $\mathrm{O} V$ ) in excellent agreement with DFT. The overall good agreement for all considered defect structures provides confidence that the $\mathrm{ABOP}$ can also describe the correct clustering behaviour of larger oxygen-based defect structures in bcc iron.

3.2.2. Oxygen adsorption on $\alpha$-Fe surfaces Adsorption energies for oxygen atoms on bcc iron (100) and (110) surfaces were calculated with the ABOP and compared with published DFT results. The common adsorption sites on (100) and (110) bcc surfaces are illustrated in figure 6. The adsorption energy is defined as

$E_{\mathrm{ad}}=E_{\mathrm{s}+\mathrm{a}}-E_{\mathrm{s}}-E_{0}$

where $E_{\mathrm{s}+\mathrm{a}}$ is the total energy of the surface slab with the adsorbate, $E_{\mathrm{s}}$ is the energy of the clean surface slab, and $E_{0}$ the energy of the isolated adsorbate in its ground state (an $\mathrm{O}_{2}$ molecule with $E_{\text {coh }}=$ $-2.583 \mathrm{eV} /$ atom in the $\mathrm{ABOP}$ ). A more negative adsorption energy represents a stronger adsorption. The surface slabs with and without adsorbates were constructed with the Fe lattice constant of the ABOP at $0 \mathrm{~K}$ and relaxed in a fixed box volume to retain the surface tension. Vacuum layers above the surfaces allowed the surface to relax in the surface normal direction, and the adsorbates to move in all dimensions.

Table 4 shows the adsorption energies predicted by the $\mathrm{ABOP}$ and compared to values from $\mathrm{DFT}$ studies [8, 9, 10]. For the (100) surface, the adsorption energy of the favoured site (hollow) is in good agreement with the DFT literature. However, the differences to the less favoured bridge and on-top sites are slightly overestimated. This is also evident in the relaxed distances between the adsorbate and the top Fe layer, i.e. the hollow site is relaxed closer to the surface, and the bridge and on-top sites further away from the surface than in the DFT results, leading to less negative adsorption energies. For the (110) surface, the strongest adsorption sites according to the DFT data are the 3-fold-hollow and the long-bridge sites (although there seems to be some uncertainty in the literature regarding which site is favoured [58]). In the ABOP, the oxygen atom relaxes towards the 3 -foldhollow site in all cases. In the on-top case, the oxygen atom moves to a position between the original site and (almost to) the 3-fold-hollow site, with an adsorption energy even slightly lower than for the 3 -fold-hollow site.

3.2.3. $\mathrm{FeO}$ properties The melting point, surface energies of the (100) and (110) surfaces, and the phonon dispersion of $\mathrm{FeO}$ (wüstite) were calculated with the ABOP. The melting point was determined 
Table 4. Adsorption energies and distances between the adsorbate and the top surface layer for oxygen atoms on the (100) and (110) $\alpha$-Fe surfaces as calculated with the ABOP and compared with published DFT results. The adsorption sites are illustrated in figure 6

\begin{tabular}{|c|c|c|c|c|}
\hline & \multicolumn{2}{|c|}{ DFT } & \multicolumn{2}{|c|}{ ABOP } \\
\hline & $E_{\text {ad }}(\mathrm{eV})$ & $d(\AA)$ & $E_{\mathrm{ad}}(\mathrm{eV})$ & $d(\AA)$ \\
\hline$(100)$ & \multicolumn{2}{|c|}{ Ref. [9] and [8] } & & \\
\hline Hollow & $-3.95,-3.72$ & $0.59,0.63$ & -3.64 & 0.33 \\
\hline Bridge & $-3.30,-3.16$ & $1.43,1.32$ & -2.72 & 1.46 \\
\hline On-top & $-2.62,-2.19$ & $1.61,1.67$ & -1.85 & 1.80 \\
\hline$(110)$ & \multicolumn{2}{|c|}{ Ref. [10] and [8] } & & \\
\hline 3-fold-hollow & -3.43, unstable & 1.05 & -2.49 & 1.25 \\
\hline Longbridge & $-3.43,-3.69$ & $1.04,1.03$ & \multicolumn{2}{|c|}{ unstable ${ }^{\mathrm{a}}$} \\
\hline Shortbridge & -2.94 , unstable & 1.25 & \multicolumn{2}{|c|}{ unstable $^{\mathrm{a}}$} \\
\hline On-top & $-1.77,-2.03$ & $1.65,1.69$ & \multicolumn{2}{|c|}{ unstable $^{\mathrm{b}}$} \\
\hline
\end{tabular}
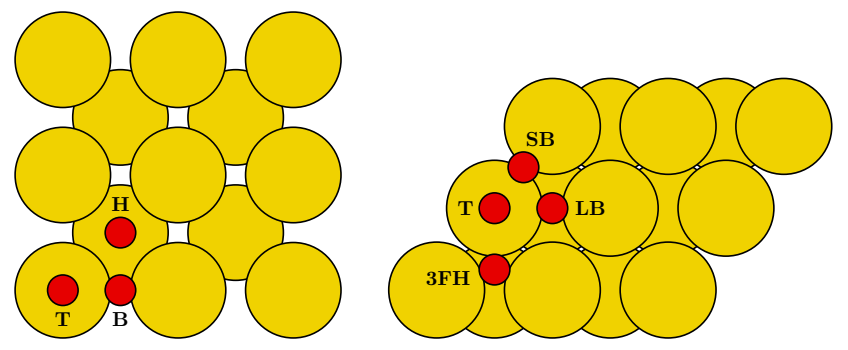

Figure 6. Common adsorption sites on a (100) bcc surface (left) and a (110) surface (right). The abbreviations marked next to each site stand for the conventional naming of the sites; on-top (T), hollow (H), bridge (B), long-bridge (LB), short-bridge (SB), and three-fold hollow $(3 \mathrm{FH})$.

by creating a solid-liquid interface and relaxing the system at different temperatures in NPT $(P=0) \mathrm{MD}$ simulations. The melting point can be estimated as the temperature at which neither the solid or liquid phase grows. At $T=1350 \mathrm{~K}$, the liquid phase is still quickly crystallising, while at $T=1450 \mathrm{~K}$ the liquid phase grows. At $T=1400 \mathrm{~K}$, the phases are in equilibrium for up to $100 \mathrm{ps}$, after which the liquid phase starts slowly growing. The melting point can then be estimated to be $1400 \pm 50 \mathrm{~K}$, in reasonable agreement with the experimental value $1644 \mathrm{~K}$ [43]. It should be noted that the experimental values are for non-stoichiometric $\mathrm{Fe}_{1-x} \mathrm{O}$, and that the trend going towards stoichiometric $\mathrm{FeO}$ is a decrease in the melting temperature 43 .

The surface energies of $\mathrm{FeO}$ in the $\mathrm{ABOP}$ are $25 \mathrm{meV} / \AA^{2}$ for the (100) surface and $34 \mathrm{meV} / \AA^{2}$ for the (110) surface. These energies are lower than the

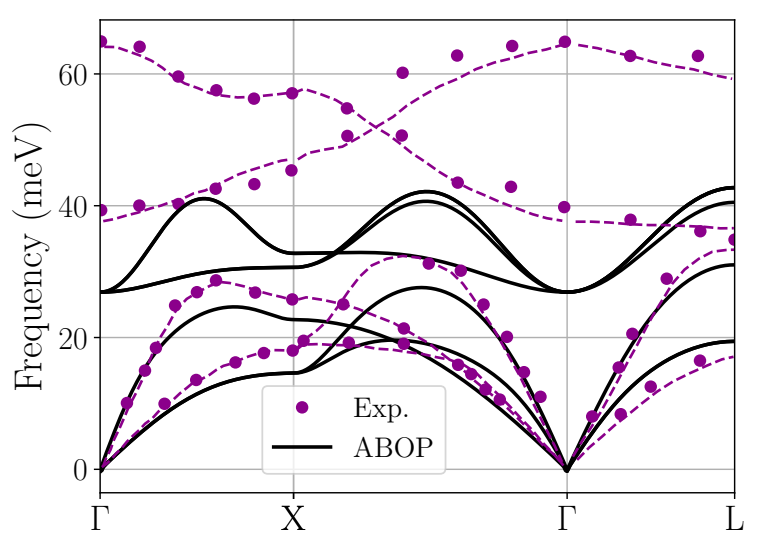

Figure 7. The phonon dispersion relation of $\mathrm{FeO}$ calculated with the ABOP and compared with experimental results. The points are experimental data, and the dashed lines results of shell-model calculations 60 .

values $61 \mathrm{meV} / \AA^{2}$ and $109 \mathrm{meV} / \AA^{2}$ obtained in the DFT study by Liao and Carter [59]. Experimental data is not available.

Figure 7 shows the phonon dispersion of $\mathrm{FeO}$ calculated with the ABOP and compared with experimental measurements and theoretical shellmodel calculations [60. The acoustical branches are in good agreement with the experimental data. The ABOP also correctly reproduces the splitting of the acoustical branches between the $\mathrm{X}$ and $\Gamma$ points seen in the shell-model calculations. However, the ABOP fails to reproduce the splitting of the longitudinal 


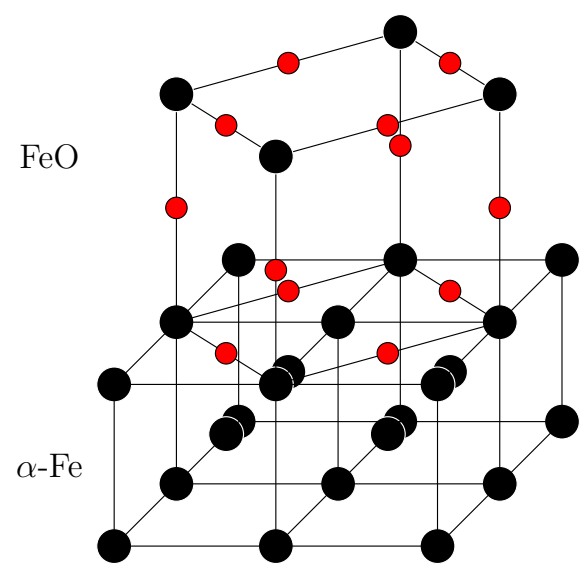

Figure 8. The interface structure between $\mathrm{FeO}$ and $\alpha$-Fe according to the Baker-Nutting orientation relationship [62].

and transverse optical branches near the $\Gamma$-point (LO-TO splitting). This is expected, as the LOTO splitting is attributed to long-range Coulombic interactions between ions in polar materials 61, which are neglected in the ABOP formalism. The frequencies of the optical branches are also clearly underestimated. This is similar to previous observations in two other ABOPs for the oxides $\mathrm{ZnO}$ [14] and $\mathrm{BeO}$ [37, and is likely also due to the lack of long-range interactions.

\subsection{Applications}

3.3.1. Thermal stability of the $\mathrm{Fe} / \mathrm{FeO}$ interface Oxidation of iron surfaces at high temperatures is known to result in an inner $\mathrm{FeO}$ layer growing on top of the iron bulk, with more oxygen-rich $\mathrm{Fe}_{3} \mathrm{O}_{4}$ and $\mathrm{Fe}_{2} \mathrm{O}_{3}$ outer layers 63. Interfaces between bcc and $\mathrm{NaCl}$ structures are known to generally obey the Baker-Nutting relationship [62; $(100)_{\mathrm{NaCl}} \|(100)_{\mathrm{bcc}}$, $[011]_{\mathrm{NaCl}} \|[001]_{\mathrm{bcc}}$, i.e. an interface where (100) surfaces of the two structures are joined with one structure rotated $45^{\circ}$ around the interface normal relative to the other, as illustrated in figure 8 . For an $\mathrm{Fe} / \mathrm{FeO}$ interface, this corresponds to a (100) $\alpha$-Fe surface with the hollow sites (see figure 6) filled with oxygen atoms, and the bcc corner atoms (the $\sqrt{2} a_{\mathrm{Fe}}$ diagonal) making up a side length of the first $\mathrm{FeO}$ unit cell, so that the interface Fe layer is shared between the two structures. This is in line with experimental and theoretical studies of the initial stages of oxidation of (100) Fe surfaces, where the hollow adsorption sites are filled and the first 1-2 monolayers of oxygen form an FeO-like thin film [58, 64. Typically, the mismatch between the lattice parameters (i.e. $\sqrt{2} a_{\mathrm{Fe}}-a_{\mathrm{FeO}}$ ) in a Baker-Nutting-type interface will result in regularly spaced misfit dislocations along the interface plane [65].

We studied an $\mathrm{Fe} / \mathrm{FeO}$ interface in MD simulations using the developed potential. The interface was
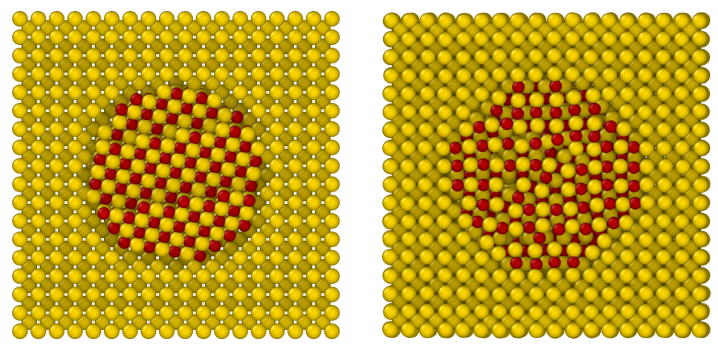

Figure 9. Cross sections of the initially spherical and the final polyhedral shape of a $3 \mathrm{~nm} \mathrm{FeO}$ precipitate in $\alpha$-Fe, as a result of annealing simulations. Red atoms are oxygen, yellow atoms iron. Note that part of the surrounding Fe bulk was cut out for better visualisation of the precipitate, and the size of the simulation system was much larger than that shown here.

constructed according to the Baker-Nutting relation. The size of the system was chosen as 15 conventional bcc Fe unit cells in each direction, joined along the $x$ axis with a rotated similarly sized $\mathrm{FeO}$ slab, 14 unit cells wide along the Fe $y z$ diagonal and 7 unit cells thick. The choice of size corresponds to a one unit cell mismatch between the structures. The Fe structure was slightly stretched in $y$ and $z$ to match the lattice spacing of the $\mathrm{FeO}$ structure, resulting in a coherent interface apart from two misfit dislocations along the edges of the interface plane. Periodic boundary conditions were used in all dimensions. Following an energy and pressure minimisation, the system was annealed for 2 ns at two different temperatures (300 $\mathrm{K}$ and $900 \mathrm{~K}$ ) and zero pressure, and subsequently cooled down to $10 \mathrm{~K}$ over $0.4 \mathrm{~ns}$. The higher annealing temperature was chosen to be below the bcc-fcc phase transition temperature, predicted by the $\mathrm{Fe} \mathrm{ABOP}$ at around $1030 \mathrm{~K}[12$.

At both temperatures, the interface retained a stable Baker-Nutting orientation throughout the simulations. At $300 \mathrm{~K}$, the final relaxed interface remained in a configuration close to the initial unrelaxed interface. The initial strains in the $y z$ plane at the interface, required for the structures to align, were evened out between the structures in the relaxed interface. The initially stretched Fe slab was slightly contracted and the $\mathrm{FeO}$ layers close to the interface compressed accordingly. The relaxed misfit dislocations along the edges in the $y z$ plane, due to the lattice mismatch, induced a significant elastic strain field throughout the system. At $900 \mathrm{~K}$, part of the oxygen atoms in the interface layer migrated one layer into the Fe slab, making the interface layers oxygendeficient and the interface and misfit dislocations less well-defined, but still oriented according the initial Baker-Nutting orientation.

3.3.2. $\mathrm{FeO}$ precipitates in $\alpha-F e$ Formation of stable $\mathrm{FeO}$ precipitates has been experimentally observed 


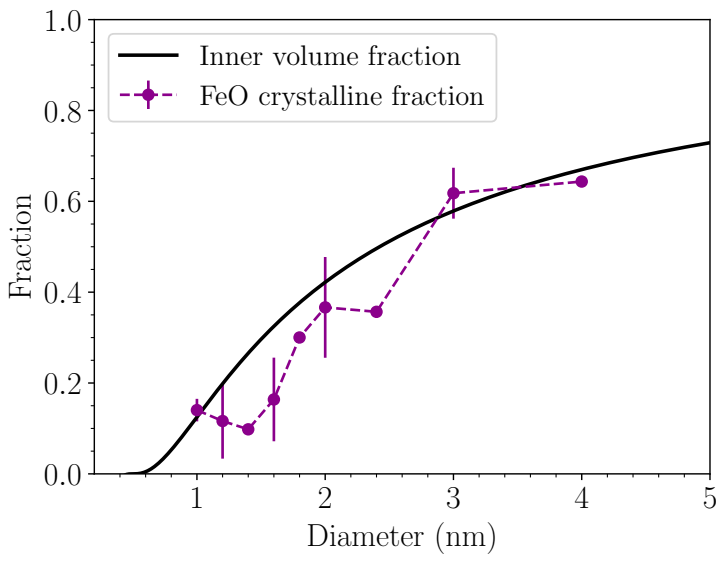

Figure 10. Crystalline fractions of the annealed $\mathrm{FeO}$ precipitates as a function of diameter. Each data point is the average of three simulations runs (except for the $4 \mathrm{~nm}$ precipitate, for which only one simulation was performed). The error bars are the standard deviations. The data is compared to the inner volume fraction of a sphere with an outer shell with a thickness of $2.5 \AA$.

in proposed methods for producing ODS steels by dissolution of iron oxides with a steel matrix 66, 67. Additionally, although the crystal structures of different oxide nanoparticles in ODS steels are still debated [4, some oxide particles have been reported to have the cubic rocksalt structure 68. As a model system, we therefore investigated the stability and shape of nano-sized $\mathrm{FeO}$ precipitates in the rocksalt structure, embedded in $\alpha$-Fe, using the ABOP. Spherical $\mathrm{FeO}$ nanoclusters with diameters from 1 to 4 $\mathrm{nm}$ were embedded in a bulk of bcc Fe by cutting out a void of the given diameter, and inserting the slightly compressed and randomly rotated $\mathrm{FeO}$ cluster 69. The surrounding Fe bulk was made large enough to ensure that the relaxed stress field induced by the $\mathrm{FeO}$ precipitate did not cause significant self-interaction effects across the periodic boundaries. The side length of the simulation system with the precipitate was 7.2 $\mathrm{nm}$ for the 1-2.4 nm precipitates, $11.6 \mathrm{~nm}$ for the 3 $\mathrm{nm}$ precipitate, and $14.4 \mathrm{~nm}$ for the $4 \mathrm{~nm}$ precipitate. After an initial energy minimisation, the systems were annealed at $900 \mathrm{~K}$ and zero pressure for $2 \mathrm{~ns}$, and subsequently cooled down to room temperature over a period of $0.2 \mathrm{~ns}$. Three separate cases with different random rotations were simulated for all sizes except the $4 \mathrm{~nm}$ precipitate, to study possible effects of the initial orientation of the precipitate. For the largest diameter $(4 \mathrm{~nm})$, only one case was simulated.

The initially spherical precipitates were seen to transform into a polyhedral shape during annealing for precipitate sizes $3 \mathrm{~nm}$ and below. The polyhedra retained the rocksalt structure in differently oriented grains, in a way that the majority of the $\mathrm{FeO} / \mathrm{Fe}$ interfaces obeyed the Baker-Nutting relationship. All simulated $3 \mathrm{~nm}$ cases resulted in similar polyhedra. Cross sections of the initial and final shape of a $3 \mathrm{~nm}$ precipitate are shown in figure 9. The 4 $\mathrm{nm}$ precipitate did not transform into a polyhedral shape, but retained its spherical shape throughout the annealing simulation. However, it is not clear whether the annealing time was too short for the transformation to take place, or if a critical size limit for the relative stability between the polyhedral and spherical $\mathrm{FeO}$ precipitates was reached. The formation of BakerNutting-type interfaces for $\mathrm{FeO}$ precipitates reaffirms the ABOP's ability to favour Baker-Nutting interfaces over other configurations, as is generally expected for interfaces between rocksalt and bcc structures 62.

Figure 10 shows the fractions of precipitate atoms in the crystalline rocksalt structure after the annealing simulations. Each data point is the average of the three simulation runs. The crystal structures were identified using ovito [70. We compare the data to the inner volume fraction of a sphere with an outer shell with the thickness $2.5 \AA$. This roughly corresponds to a perfectly crystalline precipitate with a $2.5 \AA$ interface layer of unidentifiable crystal structure, and is a good description of the $4 \mathrm{~nm} \mathrm{FeO}$ precipitate (and presumably also larger precipitates), as seen in figure 10. However, at diameters below $4 \mathrm{~nm}$, the annealed precipitates are not spherical and the interface thickness is not uniform, due to the formation of coherent Baker-Nutting interfaces. Below $4 \mathrm{~nm}$, the inner volume fraction curve therefore only serves as a guide for the expected maximum crystalline fraction. The $3 \mathrm{~nm}$ precipitates exceeds the expected perfect crystalline fraction, due to the coherent interfaces seen in figure 9, and the majority of the non-crystalline atoms are along grain boundaries inside the $\mathrm{FeO}$ cluster.

Figure 10 shows that the $\mathrm{FeO}$ precipitates never become fully amorphous. Even $1 \mathrm{~nm}$ precipitates (consisting of only 57 atoms) are partly crystalline and form small coherent Baker-Nutting interfaces. We note, however, that the majority of the simulated 1$2 \mathrm{~nm}$ precipitates do not form polyhedral clusters as symmetrical as the $3 \mathrm{~nm}$ case shown in figure 9 due to the smaller size, but are instead partly amorphous with crystalline Baker-Nutting interfaces only in some directions. The observation of nanoclusters embedded in a solid matrix becoming partly or fully amorphous is well in line with previous works [69, 71]. However, in contrast to cases with an amorphous matrix, in the current case the crystalline surrounding stabilises the nanocluster and prevents full amorphization. Our results suggest that the stability and formation of well-defined interfaces strongly affects the shape and crystallinity of oxide particles in iron. 


\section{Conclusions}

We have parametrised an interatomic potential for Fe$\mathrm{O}$ within the analytical bond-order formalism. The potential describes the energetics and geometries of oxygen interstitial atoms in $\alpha$-Fe in good agreement with density functional theory. Furthermore, the potential correctly captures the strong trapping effect of oxygen interstitials by single vacancies in iron, and fairly accurately reproduces the binding energies of different oxygen-vacancy defect clusters in $\alpha$-Fe. The potential is therefore suited for large-scale simulations of the mobility and clustering of oxygen in $\alpha$-Fe. The basic properties of the wüstite iron oxide phase $(\mathrm{FeO})$ are reproduced by the potential. We further tested the potential by calculating adsorption energies for oxygen atoms on the low-index $\alpha$-Fe surfaces, as well as the melting point, surface energies, and the phonon dispersion of wüstite. The overall agreement with experimental and ab initio data for the tested properties is good.

We applied the potential in molecular dynamics simulations of the stability of the $\mathrm{Fe} / \mathrm{FeO}$ interface, and found that the potential favours a Baker-Nutting-type interface structure, as is typical for interfaces between rocksalt and bcc crystals. Additionally, as a simplified model system of an ODS steel, we investigated the stability and shape of nano-sized $\mathrm{FeO}$ precipitates in $\alpha$-Fe. Small spherical $\mathrm{FeO}$ precipitates transformed into polyhedral shapes during annealing, driven by the formation of stable Baker-Nutting-type interfaces between the $\mathrm{FeO}$ nanoparticle and the surrounding $\alpha$ Fe bulk.

\section{Acknowledgements}

The research has been carried out with financial support from the Academy of Finland (project MIMOSE, Contract No. 259249). This work has been carried out within the framework of the EUROfusion Consortium and has received funding from the Euratom research and training programme 2014-2018 under grant agreement No 633053. The views and opinions expressed herein do not necessarily reflect those of the European Commission. Grants of computer capacity from CSC - IT Center for Science, Finland, as well as from the Finnish Grid and Cloud Infrastructure (persistent identifier urn:nbn:fi:researchinfras-2016072533) are gratefully acknowledged.

\section{References}

[1] Odette G R, Alinger M J and Wirth B D 2008 Annu. Rev. Mater. Res. 38 471-503

[2] Odette G R 2014 JOM 66 2427-2441 ISSN 1047-4838, $1543-1851$
[3] Williams C A, Unifantowicz P, Baluc N, Smith G D W and Marquis E A 2013 Acta Mater. 61 2219-2235 ISSN 1359-6454

[4] Wu Y, Ciston J, Kräemer S, Bailey N, Odette G R and Hosemann P 2016 Acta Mater. 111 108-115 ISSN 13596454

[5] Barouh C, Schuler T, Fu C C and Nastar M 2014 Phys. Rev. B 90 ISSN 1098-0121, 1550-235X

[6] Barouh C, Schuler T, Fu C C and Jourdan T 2015 Phys. Rev. B 92104102

[7] Claisse A and Olsson P 2013 Nucl. Instrum. Methods Phys. Res., Sect. B 303 18-22 ISSN 0168-583X

[8] Eder M, Terakura K and Hafner J 2001 Phys. Rev. B 64 115426

[9] Freitas R R Q, Rivelino R, de Brito Mota F and de Castilho C M C 2012 J. Phys. Chem. C 116 20306-20314 ISSN 1932-7447

[10] Liu S, Tian X, Wang T, Wen X, Li Y W, Wang J and Jiao H 2015 J. Phys. Chem. C 119 11714-11724 ISSN 1932-7447

[11] Aryanpour M, van Duin A C T and Kubicki J D $2010 \mathrm{~J}$. Phys. Chem. A 114 6298-6307 ISSN 1089-5639

[12] Müller M, Erhart P and Albe K 2007 J. Phys.: Condens. Matter 19326220 ISSN 0953-8984

[13] Björkas C and Nordlund K 2007 Nucl. Instrum. Methods Phys. Res., Sect. B 259 853-860 ISSN 0168-583X

[14] Erhart P, Juslin N, Goy O, Nordlund K, Müller R and Albe K 2006 J. Phys.: Condens. Matter 186585 ISSN 09538984

[15] Byggmästar J, Granberg F, Kuronen A, Nordlund K and Henriksson K O E 2015 J. Appl. Phys. 117014313 ISSN 0021-8979, 1089-7550

[16] Henriksson K O E, Björkas C and Nordlund K $2013 \mathrm{~J}$. Phys.: Condens. Matter 25445401 ISSN 0953-8984

[17] Müller M, Erhart P and Albe K 2007 Phys. Rev. B 76 155412

[18] Kuopanportti P, Hayward E, Fu C C, Kuronen A and Nordlund K 2016 Comput. Mater. Sci. 111 525-531 ISSN 0927-0256

[19] Mock M and Albe K 2018 J. Nucl. Mater. 509 102-113 ISSN 0022-3115

[20] Henriksson K O E 2013 TULIP: Program for fitting an interatomic potential to data

[21] Plimpton S 1995 J. Comput. Phys. 117 1-19 ISSN 00219991 http://lammps.sandia.gov

[22] Nordlund K, Ghaly M, Averback R S, Caturla M, Diaz de la Rubia T and Tarus J 1998 Phys. Rev. B 57 7556-7570

[23] Ghaly M, Nordlund K and Averback R S 1999 Philos. Mag. A 79 795-820 ISSN 0141-8610

[24] Larsen A H, Mortensen J J, Blomqvist J, Castelli I E, Christensen R, Marcin Dułak, Friis J, Groves M N, Hammer B, Hargus C, Hermes E D, Jennings P C, Jensen P B, Kermode J, Kitchin J R, Kolsbjerg E L, Kubal J, Kristen Kaasbjerg, Lysgaard S, Maronsson J B, Maxson T, Olsen T, Pastewka L, Andrew Peterson, Rostgaard C, Schiøtz J, Schütt O, Strange M, Thygesen K S, Tejs Vegge, Vilhelmsen L, Walter M, Zeng Z and Jacobsen K W 2017 J. Phys.: Condens. Matter 29273002 ISSN 0953-8984

[25] Kresse G and Furthmüller J 1996 Comput. Mater. Sci. 6 15-50 ISSN 0927-0256

[26] Blöchl P E 1994 Phys. Rev. B 50 17953-17979

[27] Perdew J P, Burke K and Ernzerhof M 1996 Phys. Rev. Lett. $773865-3868$

[28] Abell G C 1985 Phys. Rev. B 31 6184-6196

[29] Tersoff J 1986 Phys. Rev. Lett. 56 632-635

[30] Tersoff J 1988 Phys. Rev. B 37 6991-7000

[31] Tersoff J 1988 Phys. Rev. B 38 9902-9905

[32] Tersoff J 1988 Phys. Rev. Lett. 61 2879-2882

[33] Brenner D W 1990 Phys. Rev. B 42 9458-9471 
[34] Brenner D W 1989 Phys. Rev. Lett. 63 1022-1022

[35] Daw M S and Baskes M I 1984 Phys. Rev. B $296443-6453$

[36] Foiles S M, Baskes M I and Daw M S 1986 Phys. Rev. B 33 7983-7991

[37] Byggmästar J, Hodille E A, Ferro Y and Nordlund K 2018 J. Phys.: Condens. Matter 30135001 ISSN 0953-8984

[38] Albe K, Nordlund K and Averback R S 2002 Phys. Rev. B 65195124

[39] Byggmästar J 2016 Development of interatomic potentials in the Tersoff-Albe formalism for metal compounds MSc thesis, http://hdl.handle.net/10138/166468

[40] Mrovec M, Nguyen-Manh D, Elsässer C and Gumbsch P 2011 Phys. Rev. Lett. 106246402

[41] Lin Y S, Mrovec M and Vitek V 2016 Phys. Rev. B 93 ISSN 2469-9950, 2469-9969

[42] Ziegler J F, Biersack J P and Littmarck U 1985 The Stopping and Range of Ions in Matter Treatise on HeavyIon Science (New York: Pergamon) pp 93-129 ISBN 9781-4615-8105-5 978-1-4615-8103-1

[43] Predel B 1995 Fe-O (Iron-Oxygen) Dy-Er - Fr-Mo (Landolt-Börnstein - Group IV Physical Chemistry no 5e) ed Madelung O (Springer Berlin Heidelberg) pp 1-5 ISBN 978-3-540-58428-5 978-3-540-48786-9

[44] Cornell R M and Schwertmann U 2003 The Iron Oxides: Structure, Properties, Reactions, Occurrences and Uses 2nd ed (Darmstadt: John Wiley \& Sons) ISBN 978-3527-30274-1

[45] Zhang J 2000 Phys. Rev. Lett. 84 507-510

[46] Isaak D and Moser S 2013 J. Phys. Chem. Solids 74 879885 ISSN 0022-3697

[47] Wright J P, Attfield J P and Radaelli P G 2002 Phys. Rev. B 66214422

[48] Reichmann H J and Jacobsen S D 2004 Am. Mineral. 89 1061-1066

[49] Finger L W and Hazen R M 1980 J. Appl. Phys. 51 53625367 ISSN 0021-8979, 1089-7550

[50] Wilburn D R, Bassett W A, Sato Y and Akimoto S $1978 \mathrm{~J}$. Geophys. Res. 83 3509-3512 ISSN 2156-2202

[51] Sato Y and Akimoto S i 1979 J. Appl. Phys. 50 5285-5291 ISSN 0021-8979, 1089-7550

[52] Fu C L, Krčmar M, Painter G S and Chen X Q 2007 Phys. Rev. Lett. 99225502

[53] Shang S L, Fang H Z, Wang J, Guo C P, Wang Y, Jablonski P D, Du Y and Liu Z K 2014 Corros. Sci. 83 94-102 ISSN 0010-938X

[54] Jiang Y, Smith J R and Odette G R 2009 Phys. Rev. B 79 064103

[55] Metz R B, Nicolas C, Ahmed M and Leone S R $2005 \mathrm{~J}$. Chem. Phys. 123114313 ISSN 0021-9606, 1089-7690

[56] Haynes W M 2015 CRC Handbook of Chemistry and Physics 96th ed (Boca Raton, FL: CRC Press) ISBN 978-1-4822-6097-7

[57] Murali D, Panigrahi B K, Valsakumar M C, Chandra S, Sundar C S and Raj B 2010 J. Nucl. Mater. 403 113116 ISSN 0022-3115

[58] Parkinson G S 2016 Surf. Sci. Rep. 71 272-365 ISSN 01675729

[59] Liao P and Carter E A 2010 J. Mater. Chem. 20 6703-6719 ISSN 1364-5501

[60] Kugel G, Carabatos C, Hennion B, Prevot B, Revcolevschi A and Tocchetti D 1977 Phys. Rev. B 16 378-385

[61] Ashcroft N W and Mermin N D 1976 Solid State Physics (Philadelphia: Saunders College Publishing)

[62] Baker R and Nutting J 1959 ISI Spec. Rep. 64

[63] Chen R Y and Yeun W Y D 2003 Oxidation of Metals 59 433-468 ISSN 0030-770X, 1573-4889

[64] Błoński P, Kiejna A and Hafner J 2005 Surf. Sci. 590 88100 ISSN 0039-6028

[65] Fors D H R, Johansson S A E, Petisme M V G and Wahnström G 2010 Comput. Mater. Sci. 50 550-559
ISSN 0927-0256

[66] Sagaradze V V, Litvinov A V, Shabashov V A, Vil'danova N F, Mukoseev A G and Kozlov K A 2006 Phys. Metals Metallogr. 101 566-576 ISSN 0031-918X, 1555-6190

[67] Sagaradze V V, Kozlov K A, Kataeva N V, Litvinov A V and Shabashov V A 2012 Phys. Metals Metallogr. 113 372-381 ISSN 0031-918X, 1555-6190

[68] Hirata A, Fujita T, Wen Y R, Schneibel J H, Liu C T and Chen M W 2011 Nat. Mater. 10 922-926 ISSN 1476-1122

[69] Djurabekova F and Nordlund K 2008 Phys. Rev. B 77 115325

[70] Stukowski A 2010 Modelling Simul. Mater. Sci. Eng. 18 015012 ISSN 0965-0393 http://ovito.org/

[71] Sprouster D J, Giulian R, Araujo L L, Kluth P, Johannessen B, Kirby N, Nordlund K and Ridgway M C 2010 Phys. Rev. B 81155414 